\title{
The analitical modeling of the motion process of the inverted pendulum system
}

\author{
Pavel Obukhov ${ }^{1, *}$, Yaroslav Mogilin² \\ ${ }^{1}$ Department of automation of production processes, Don State Technical University, Russia \\ ${ }^{2}$ Department of automation of production processes, Don State Technical University, Russia
}

\begin{abstract}
In the article the conceptual description of the nonlinear mathematical model of the inverted pendulum is presented. The Lagrange's equations of the second kind are applying to build of the model, the generalized coordinate are selected and the system of the differential equations of the fourth odder is formed.
\end{abstract}

\section{Introduction}

The problem of the building of the systems of the automatic control process of the object movement type the inverted pendulum is urgent and very complex. These systems widespread in practice, when the large objects with a high center of the gravity are moving. The mathematical modeling play important role in the synthesis of the such control system by which it is possible to abstract from the real variables and to efficient explore the problem. The quality of the system control functioning ultimately depends of the accuracy of the construction of the control object model.

\section{Main part}

The parameters of the inverted pendulum necessary to identify for the conceptual description of the control object. The transport complex which consist of the platform of mass $\mathrm{m} 2$ which is moving on rails or surface (horizontal) and the vertical rod of mass $\mathrm{m} 1$ and length $\mathrm{L}$ are attached to the platform by using of the electromechanically device (without signal control it works such as hinge) is presented on the figure 1 . The rod is deviate from the balance position at the angel $\varphi$ by the disturbance (wind flow $\mathrm{G}=\mathrm{F}_{\mathrm{B}} / \mathrm{L}$ ) and the force of gravity $\mathrm{P}$ acts at the center of the gravity $P_{L T}=\frac{2 L}{3}$. The thrust force $\mathrm{Fu}$ applied to the platform for the motion in the horizontal direction are used. The moment turns the rod at the certain angel for its return to the balance position.

With compiling the mathematical model (MM) of the control object $(\mathrm{CO})$ as a control actions are taken the force $\mathrm{Fu}$ that causes the platform to move at the horizontally direction and the moment $\mathrm{Mu}$ are rotated the rod at the certain angel. As the disturbance we will take the wind which acts on the all surface along the length of the rod is considered in the following as a stream.
Some assumptions to obtain the MM OC: the parameters of the $\mathrm{OC}$ are concentrated (the rod and the platform are uncompressible objects); the platform moves on rails without friction and only at the horizontally direction; the rod rotates on the hinge mechanism without friction; the cylinder is forming a shape of the rod, the moment of the inertia of the cylinder are considered.

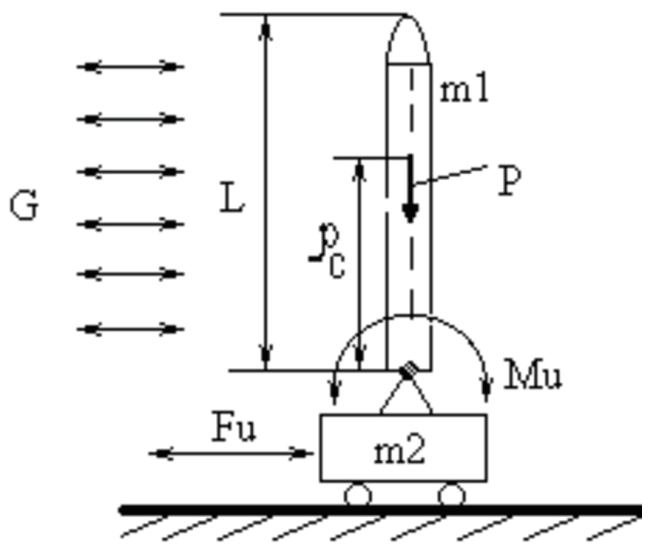

Fig. 1. The transport complex

The Lagrange's equations of the second kind are applying to build of the MM, for this the generalized coordinates are selected, in this case it may be the motion of the platform at the horizontally direction $\mathrm{X}$ and the deviate angel of the rod at the balance position $\varphi(1)$,

$$
\left\{\begin{array}{l}
\mathrm{q}_{1}=X \\
\mathrm{q}_{2}=\varphi
\end{array}\right.
$$

then $\delta \mathrm{X}$ - the variation of the displacement for a given deviation, $\delta \varphi$ - the variations of the rotate angel of the rod (see figure 2).

\footnotetext{
* Corresponding author: pobuhov@spark-mail.ru
} 


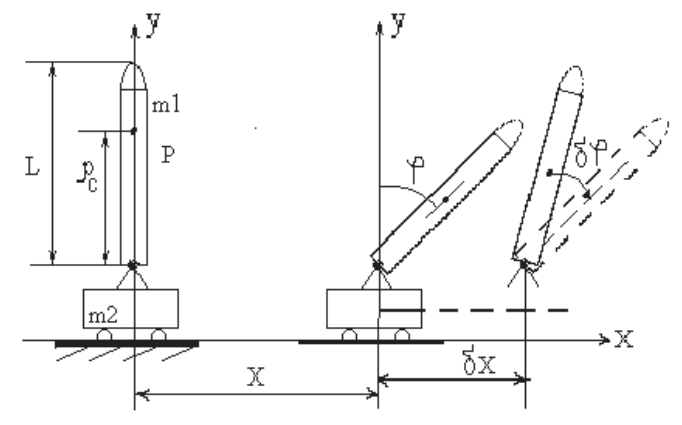

Fig. 2. The choice of the generalized coordinates

The equations system with the generalized coordinates chosen takes the form:

$$
\left\{\begin{array}{l}
\frac{\mathrm{d}}{\mathrm{dt}}\left(\frac{\partial \Gamma}{\partial \dot{\mathrm{X}}}\right)-\frac{\partial \mathrm{T}}{\partial \mathrm{X}}=\mathrm{Q}_{\mathrm{X}}-\frac{\partial \Pi}{\partial \mathrm{X}} \\
\frac{\mathrm{d}}{\mathrm{dt}}\left(\frac{\partial \mathrm{T}}{\partial \dot{\varphi}}\right)-\frac{\partial \mathrm{T}}{\partial \varphi}=\mathrm{Q}_{\varphi}-\frac{\partial \Pi}{\partial \varphi}
\end{array}\right.
$$

where $\mathrm{T}$ - kinematic energy; $\Pi$ - potential energy; $\mathrm{Q}_{\mathrm{X}}, \mathrm{Q}_{\varphi}$ - generalized forces on displacement $\mathrm{X}$ and angel of the deviation $\varphi$.

The expressions of the kinematic and potential energy of the control object and their derivatives and also expressions for the generalized forces are found by the formulas:

$$
\begin{aligned}
& \frac{\partial T}{\partial X}=0, \text { T.K. T }=T(\varphi, \dot{X}, \dot{\varphi}) \\
& \frac{\partial T}{\partial \varphi}=-\frac{2 m_{1}}{3} \cdot L \cdot \dot{X} \cdot \dot{\varphi} \cdot \sin \varphi \\
& \frac{\partial T}{\partial \dot{\varphi}}=\dot{\varphi} \cdot \frac{m_{1} \cdot L^{2} \cdot 7}{9}+\frac{2 m_{1} \cdot L}{3} \cdot \dot{X} \cdot \cos \varphi \\
& \frac{\partial T}{\partial \dot{X}}=\dot{X} \cdot\left(m_{1}+m_{2}\right)+\frac{2 m_{1} \cdot L}{3} \cdot \dot{\varphi} \cdot \cos \varphi \\
& \frac{d}{d t}\left(\dot{X} \cdot\left(m_{1}+m_{2}\right)+\frac{2 m_{1} \cdot L}{3} \cdot \dot{\varphi} \cdot \cos \varphi\right)= \\
& =\ddot{X} \cdot\left(m_{1}+m_{2}\right)+\frac{2 m_{1} \cdot L}{3} \cdot\left(\dot{\varphi} \cdot \cos \varphi-\dot{\varphi}^{2} \cdot \sin \varphi\right) \\
& \frac{d}{d t}\left(\dot{\varphi} \cdot \frac{m_{1} \cdot L^{2} \cdot 7}{9}+\frac{2 m_{1} \cdot L}{3} \cdot \dot{X} \cdot \cos \varphi\right)= \\
= & \ddot{\varphi} \cdot \frac{m_{1} \cdot L^{2} \cdot 7}{9}+\frac{2 m_{1} \cdot L}{3} \cdot(\ddot{X} \cdot \cos \varphi-\dot{X} \cdot \dot{\varphi} \cdot \sin \varphi) \\
Q_{X}= & G \cdot L \cdot \cos \varphi+F_{U} \\
Q_{\varphi}=G & \frac{2 L^{2}}{3} \cdot \cos ^{2} \varphi+M_{U} \\
& \frac{\partial \Pi / \partial \varphi=-m_{1} \cdot g \cdot \rho_{C} \cdot \sin \varphi}{\partial \Pi / \partial X=0} \\
&
\end{aligned}
$$

The expression is substituted into the formula (2) and obtain the system of equations:

$$
\left\{\begin{array}{c}
\ddot{X} \cdot\left(m_{1}+m_{2}\right)+\frac{2 m_{1} \cdot L}{3} \cdot\left(\ddot{\varphi} \cdot \cos \varphi-\dot{\varphi}^{2} \cdot \sin \varphi\right)=G \cdot L \cdot \cos \varphi+F_{U} \\
\ddot{\varphi} \frac{m_{1} \cdot L^{2} \cdot 7}{9}+\frac{2 m_{1} \cdot L}{3} \cdot(\ddot{X} \cdot \cos \varphi-\dot{X} \cdot \dot{\varphi} \cdot \sin \varphi)+\frac{2 m_{1}}{3} \cdot L \cdot \dot{X} \cdot \dot{\varphi} \cdot \sin \varphi=\frac{G 2 \cdot L^{2}}{3} \cdot \cos ^{2} \varphi+M_{U}+ \\
+m_{1} \cdot g \cdot \frac{2 L}{3} \cdot \sin \varphi
\end{array}\right.
$$

The system of The Lagrange's equations of the second kind descriptions the control object of study from the point of view the dynamic of the mechanical system and it is her equivalent of the mathematical model based on the assumptions made in the problem formulation.

For further research of the control object the system is converted to the form of Cauchy

$$
\begin{aligned}
& \left\{\begin{array}{c}
\dot{X}=V \\
\dot{\varphi}=\omega \\
\dot{\omega}=\frac{\frac{2 m \cdot L \cdot \omega^{2} \cdot \sin \varphi}{3 \cdot\left(m+m_{2}\right)}+\frac{G L \cdot \cos \varphi}{\left(m+m_{2}\right)}+\frac{F_{U}}{\left(m+m_{2}\right)}-\frac{G L \cdot \cos \varphi}{m_{1}}-g \cdot \operatorname{tg} \varphi-\frac{3 \cdot M_{U}}{2 m_{1} \cdot L \cdot \cos \varphi}}{\left(\frac{2 L \cdot \cos \varphi}{3 \cdot\left(1+\frac{m_{2}}{m_{1}}\right)}-\frac{7 \cdot L}{6 \cdot \cos \varphi}\right)}
\end{array}\right. \\
& \dot{V}=\frac{\operatorname{tg} \varphi \omega^{2}+\frac{G 3}{2 m_{\uparrow}}+\frac{3 \cdot F_{U}}{2 m \cdot L \cdot \cos \varphi} \frac{G 6 \cdot \cos ^{2} \varphi}{m_{\uparrow} \cdot 7} \frac{6 \cdot g \cdot \sin \varphi}{L \cdot 7} \frac{M_{U} \cdot 9}{m_{\uparrow} \cdot L^{2} \cdot 7}}{\left(\frac{\left(1+\frac{m_{2}}{m}\right) \cdot 3}{L \cdot \cos \varphi}-\frac{\cos \varphi 6}{L \cdot 7}\right)}
\end{aligned}
$$

\section{Conclusions}

Thus, the mathematical model of the inverted pendulum is the system of the equations of the first order, which contains nonlinearity of the type of the product. The next stage of the research is the task of the analyze of the mathematical model of this object and synthesis of the control system.

\section{References}

1. A. A. Kolesnikov, Synergetic control of mechanical system "inverted pendulum on a cart" nonlinear coordinate transformation, TRTU, (2001)

2. Yu. G. Martynenko, A. M. Formalsky, Reports of Academy of Sciences, 439, N6 (2011)

3. E.I. Butikov, Komp'yuternyye instrumenty v obrazovanii, № 5, 40 - 51 (2010) 